\title{
Panta rei
}

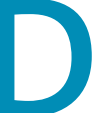
as Jahr 2004 neigt sich dem Ende zu. Es ist daher an der Zeit, sich in diesem letzten Heft des Jahres Gedanken darüber zu machen, was 2004 für allergische Patienten und ihre behandelnden Ärzte gebracht hat: Sicherlich ein nicht $\mathrm{zu}$ überbietendes gesundheitspolitisches Wirrwarr. Erst soll der EBM 2000 plus ganz sicher eingeführt werden, dann wiederum ganz sicher nicht, zwischenzeitlich dann doch wieder ...?

Ähnliches wiederholt sich mit der Erstattungsfähigkeit von Antiallergika, der Konzeption und Einführung von Disease-Management-Programmen für Asthma und Allergien, der integrierten Versorgung, der verbindlichen Einführung von DRGs als Honorierungssystem im Krankenhaus, der Novellierung der Weiterbildungsordnung - eine Liste nicht enden wollender Chaostheorien.

Panta rei - alles fließt und die einzige Konstante in dieser Gesundheitspolitik ist der stete Wandel. Verlässliche Planungen sind für alle Beteiligten unmöglich und beharrlich

„Es ist unwürdig, wie mit den Patientinnen und Patienten umgegangen wird. Denn sie sind das schwächste Glied in der Kette eines Systems, das zurzeit seinen Offenbarungseid abzulegen scheint." wird ein (doch gar

nicht so schlechtes) Gesundheitssystem demontiert, ohne ein neues etabliert zu haben.

Apothekenpflichtige, nicht verschreibungspflichtige Arzneimittel können seit dem 1. Januar nicht mehr zu Lasten der Gesetzlichen Krankenversicherung verordnet werden. Die vom gemeinsamen Bewertungsausschuss (GBA) getroffenen Ausnahmeregelungen für die Versorgung Schwerkranker sind unzureichend und schließen viele chronisch Hautkranke sowie schwer- und schwerstkranke Allergiker von einer fach- und leitliniengerechten Versorgung aus. Es ist unwürdig, wie mit den Patientinnen und Patienten umgegan- gen wird. Denn sie sind das schwächste Glied in der Kette eines Systems, das zurzeit seinen Offenbarungseid abzulegen scheint. Die indifferente dermatologische Therapie, ein Jahrzehnte altes, evident (einleuchtend) wirksames Therapieprinzip wird von selbst ernannten „Aposteln des Heils“ kurzerhand zu einer kosmetischen Maßnahme (Pflege) erklärt. Standardtherapeutika bei akuten wie auch chronischen Hauterkrankungen und Allergien sind daher nicht mehr erstattungsfähig. Es drohen nicht nur Therapieabbrüche, nein, sie finden täglich statt. Dies führt zu einer erheblichen Verschlimmerung der jeweiligen Krankheitsbilder und in der Folge zu unnötigen, wiederholten Krankenhausaufenthalten. Und dort werden Ärzte durch ständiges Nachfragen der Medizinischen Dienste der Krankenkassen in ihrer primären Aufgabe blockiert, Patienten optimal zu versorgen. Patien-

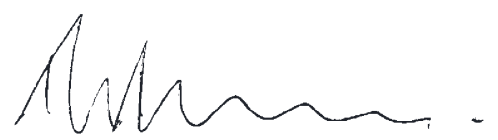

Prof. Dr. Thomas Fuchs tinnen und Patienten werden unverhohlen zu Kunden erklärt. Wir können und wollen uns nicht vorstellen, dass all dieses beabsichtigt war und ist. Oder etwa doch? Merkwürdig ist, dass viele allergische Patienten den Weg in die Praxis des Allergologen nicht mehr oder erst spät, in manchen Fällen zu spät finden. Die gemeinsamen Bemühungen von ÄDA, DGAI, DDG und BVDD sowie den Patientenverbänden hinsichtlich einer sachdienlicheren Auslegung der Ausnahmeregelungen haben bislang keine Wirkung gezeigt. Hier hoffen wir auf späte Einsicht in 2005.

Das Jahr 2004 hat uns aber auch unvergleichliche positive Eindrücke gebracht. So zum Beispiel die gemeinsame Jahrestagung der allergologischen Gesellschaften ÄDA, DGAI und GPA in Aachen, die es in dieser Form noch nie zuvor gegeben hat. Die Tagung weist in die richtige Richtung. Wie heißt es so schön: Nur gemeinsam sind wir stark. Die fachlichen Bereicherungen und auch die zwischenmenschlichen Begegnungen werden sicherlich in sehr angenehmer Erinnerung bleiben. Den beiden Organisatoren Hans Merk und Frank Friedrichs sei an dieser Stelle noch einmal herzlich dafür gedankt.

Es bleibt zu hoffen, dass wir im Jahr 2005 trotz der gesundheitspolitischen Stürme weiterhin zum Wohle der uns anvertrauten Patientinnen und Patienten tätig sein können.

Wir wünschen Ihnen und Ihren Familien eine geruhsame Adventszeit, glückliche Weihnachtstage und alles Gute für 2005!
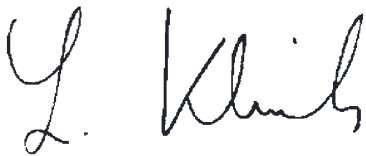

Prof. Dr. Ludger Klimek 